KIAS-P07040

\title{
Charged Black Holes on DGP Brane
}

\author{
Ee Chang-Young ${ }^{\dagger, \pm 11}$ and Daeho LeE ${ }^{\dagger} 2_{2}$ \\ ${ }^{\dagger}$ Department of Physics, Sejong University, Seoul 143-747, Korea \\ $\ddagger$ School of Physics, Korea Institute for Advanced Study, Seoul 130-722, Korea
}

\begin{abstract}
We consider charged black holes localized on a three-brane in the DGP model. With a spherically symmetric metric ansatz on the brane and assuming a $Z_{2}$-symmetry across the brane, we find two types of solutions which correspond to the so-called regular and accelerated branches in the DGP model. When the charge of the black hole vanishes, our solutions agree with the Schwarzschild solutions obtained by Gabadadze and Iglesias.
\end{abstract}

PACS: 04.40.Nr, 04.50.+h, 04.70.-S

\footnotetext{
${ }^{1}$ cylee@sejong.ac.kr

${ }^{2}$ dhlee@sju.ac.kr
} 


\section{Introduction}

Recent astronomical observations indicate that our universe is in the phase of accelerated expansion [1]. The DGP model [2] is known to contain a branch of solutions exhibiting self accelerated expansion of the universe [3].

The brane-world black holes in the case of Randall-Sundrum(RS) model [4] were first considered in [5]. Then, Dadhich et al. [6] showed that for static black holes localized on a three-brane in the RS model the Reissner-Nördstrom metric is an exact solution even when black holes are uncharged. They interpreted it as a 'tidal charge' effect arising via gravitational effect from the extra dimension. Then a solution of charged brane-world black holes in the RS model was obtained in [7], and the charged rotating case was obtained in [8]. On the other hand, for the DGP model only approximate Schwarzschild solutions had been obtained in [9, 10, 11, 12, 13] until Gabadadze and Iglesias obtain the exact Schwarzschild solutions in [14].

However, in [15] the instability issue of the static uncharged black holes on the brane was raised. The brane with the induced graviton kinetic term has effectively repulsive gravity and thus push any sources off the brane. It was argued that the charged black holes could be quasilocalized when the corresponding gauge fields are localized. Thus the need of investigating the charged case was called for in [14]. So far, the solutions of charged or rotating black holes on the brane in the DGP model have not been known.

In this paper, we investigate the solutions of charged black holes located on the threebrane in the DGP model. We solve this by assuming a spherically symmetric metric ansatz on the brane, and find two types of solutions one of which exhibits the self acceleration of the brane-world universe.

This paper is organized as follows. In section II, we set the action and equations of motion of the DGP model following the approach of Ref. [16]. In section III, we give exact solutions for the metric on the brane in the presence of the Maxwell field. In section IV, we conclude with the discussion. 


\section{Action and field equations}

The DGP gravitational action in the presence of sources takes the form [2]

$$
S=M_{*}^{3} \int d^{5} x \sqrt{-g}^{(5)} R+\int d^{4} x \sqrt{-h}\left(M_{P}^{2} R+L_{\text {matter }}\right),
$$

where $R$ and ${ }^{(5)} R$ are the 4D and 5D Ricci scalars, respectively and $L_{\text {matter }}$ is the Lagrangian of the matter fields trapped on the brane. Here, the $(4+1)$ coordinates are $x^{A}=\left(x^{\mu}, y(=\right.$ $\left.\left.x^{5}\right)\right), \mu=0,1,2,3$, and $g$ and ${ }^{(5)} R$ are the determinant and curvature of the five-dimensional metric $g_{A B}$, while $h$ and $R$ are the determinant and curvature of the four-dimensional metric $h_{\mu \nu}=g_{\mu \nu}\left(x^{\mu}, y=0\right)$. A cross-over scale is defined by $r_{c}=m_{c}^{-1}=M_{P}^{2} / 2 M_{*}^{3}$. There is a boundary(a brane) at $y=0$ and $Z_{2}$ symmetry across the boundary is assumed. The boundary Gibbons-Hawking term [17] is implied to yield the correct Einstein equations in the bulk. The field equations derived from the action (1) have the form

$$
{ }^{(5)} G_{A B}={ }^{(5)} R_{A B}-\frac{1}{2} g_{A B}{ }^{(5)} R=\kappa_{5}^{2} \sqrt{\frac{h}{g}}\left(X_{A B}+T_{A B}\right) \delta(y),
$$

where $\kappa_{4}^{2}=M_{P}^{-2}$ and $\kappa_{5}^{2}=M_{*}^{-3}$, while $X_{A B}=-\delta_{A}^{\mu} \delta_{B}^{\nu} G_{\mu \nu} / \kappa_{4}^{2}$ and $T_{A B}=\delta_{A}^{\mu} \delta_{B}^{\nu} T_{\mu \nu}$ is the energy-momentum tensor in the braneworld. Now, we consider the metric of the following form,

$$
d s^{2}=g_{A B} d x^{A} d x^{B}=g_{\mu \nu}(x, y) d x^{\mu} d x^{\nu}+2 N_{\mu} d x^{\mu} d y+\left(N^{2}+g_{\mu \nu} N^{\mu} N^{\nu}\right) d y^{2},
$$

where the so-called lapse scalar field $N$ and the shift vector field $N_{\mu}$ are defined by [16, 18]

$$
g_{\mu 5} \equiv N_{\mu}=g_{\mu \nu} N^{\nu}, g_{55} \equiv N^{2}+g_{\mu \nu} N^{\mu} N^{\nu}
$$

The $(\mu 5),(55)$ components of the field equations (2) are called as the momentum and Hamiltonian constraint equations, respectively, and are given by [16, 18]

$$
\begin{gathered}
\nabla_{\nu} K_{\mu}^{\nu}-\nabla_{\mu} K=0, \\
R-K^{2}+K_{\mu \nu} K^{\mu \nu}=0,
\end{gathered}
$$

where $K_{\mu \nu}$ is the extrinsic curvature tensor defined by

$$
K_{\mu \nu}=\frac{1}{2 N}\left(\partial_{y} g_{\mu \nu}-\nabla_{\mu} N_{\nu}-\nabla_{\nu} N_{\mu}\right)
$$


and $\nabla_{\mu}$ is the covariant derivative operator associated with the metric $g_{\mu \nu}$.

To implement the Israel's junction condition [19], we integrate both sides of the field equation (2) along the $y$ direction and take the limit of $y=0$ on the both sides of the brane:

$$
\lim _{\epsilon \rightarrow 0} \int_{-\epsilon}^{+\epsilon} d y{ }^{(5)} G_{\mu \nu}=\lim _{\epsilon \rightarrow 0} \int_{-\epsilon}^{+\epsilon} d y \kappa_{5}^{2} \sqrt{\frac{h}{g}}\left(X_{\mu \nu}+T_{\mu \nu}\right) \delta(y),
$$

where

$$
\begin{aligned}
{ }^{(5)} G_{\mu \nu}= & G_{\mu \nu}-\frac{1}{N}\left[\left(\partial_{y}-\mathcal{L}_{\vec{N}}\right)\left(K_{\mu \nu}-g_{\mu \nu} K\right)+\nabla_{\mu} \nabla_{\nu} N\right] \\
& -3 K K_{\mu \nu}+2 K_{\mu}{ }^{\rho} K_{\rho \nu}+\frac{1}{2} g_{\mu \nu}\left(K^{2}+K_{\rho \sigma} K^{\rho \sigma}+\frac{2}{N} \nabla_{\rho} \nabla^{\rho} N\right) .
\end{aligned}
$$

Here the Lie derivative $\mathcal{L}_{\vec{N}}$ is taken along the shift vector $N^{\mu}$, and the Israel's junction condition becomes,

$$
\lim _{\epsilon \rightarrow 0}\left[-\frac{1}{N}\left(K_{\mu \nu}-g_{\mu \nu} K\right)\right]_{-\epsilon}^{+\epsilon}=\kappa_{5}^{2} \sqrt{\frac{h}{g}}\left(X_{\mu \nu}+T_{\mu \nu}\right) .
$$

Using the relation $\frac{1}{N}=\sqrt{\frac{h}{g}}$, this can be written as

$$
\left[K_{\mu \nu}\right]-h_{\mu \nu}[K]=-\kappa_{5}^{2}\left(X_{\mu \nu}+T_{\mu \nu}\right)
$$

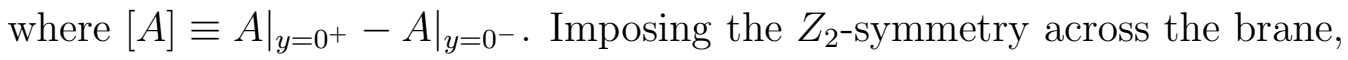

$$
K_{\mu \nu}^{+}=-K_{\mu \nu}^{-}
$$

and taking the limit $y=0$, the junction condition (11) can be expressed as

$$
G_{\mu \nu}=\kappa_{4}^{2} T_{\mu \nu}+m_{c}\left(K_{\mu \nu}-h_{\mu \nu} K\right)
$$

Once we take the electro-magnetic field as the matter source on the brane, the trace of the energy momentum tensor for the electro-magnetic field vanishes. The momentum constraint equation (5) on the brane is satisfied identically due to (13),

$$
0=D_{\mu} K_{\nu}^{\mu}-D_{\nu} K=D^{\mu}\left(K_{\mu \nu}-h_{\mu \nu} K\right)
$$

where $D_{\mu}$ is the covariant derivative operator associated with the brane metric $h_{\mu \nu}$. The Hamiltonian constraint on the brane ([6]) is given by

$$
0=R+\frac{1}{m_{c}^{2}}\left(\kappa_{4}^{4} T_{\mu \nu} T^{\mu \nu}+R_{\mu \nu} R^{\mu \nu}-\frac{1}{3} R^{2}-2 \kappa_{4}^{2} R_{\mu \nu} T^{\mu \nu}\right) .
$$


In what follows we shall set $\kappa_{4}^{2}=8 \pi$.

Finally, combining the five-dimensional Einstein Equations

$$
{ }^{(5)} G_{A B}={ }^{(5)} R_{A B}-\frac{1}{2} g_{A B}{ }^{(5)} R=0,
$$

with the Israel's junction condition (13), we arrive at the gravitational field equations on the brane

$$
G_{\mu \nu}=-E_{\mu \nu}-\kappa_{5}^{4} \tilde{\tau}_{\mu \nu}
$$

where $E_{\mu \nu}$, the traceless "eletric part" of the 5-dimensional Weyl tensor ${ }^{(5)} C_{A B C D}$, is defined by 20

$$
E_{\mu \nu}={ }^{(5)} C_{A B C D} n^{A} n^{C} e_{\mu}{ }^{B} e_{\nu}^{D}
$$

and

$$
\tilde{\tau}_{\mu \nu}=\frac{1}{4}\left[\tau_{\mu}^{\rho} \tau_{\rho \nu}-\frac{1}{3} \tau \tau_{\mu \nu}-\frac{1}{2} h_{\mu \nu}\left(\tau_{\rho \sigma} \tau^{\rho \sigma}-\frac{1}{3} \tau^{2}\right)\right],
$$

with $\tau_{\mu \nu}=T_{\mu \nu}+X_{\mu \nu}$. It should be noted that $E_{\mu \nu}$ above is the limiting value at $y=+0$ or -0 but not the value exactly on the brane. Using the relation $X_{\mu \nu}=-G_{\mu \nu} / \kappa_{4}^{2}$, the gravitational field equation (17) on the brane is given by

$$
\begin{aligned}
G_{\mu \nu}= & -E_{\mu \nu}-\frac{\kappa_{4}^{4}}{m_{c}^{2}}\left(T_{\mu}^{\rho} T_{\rho \nu}-\frac{1}{2} h_{\mu \nu} T_{\rho \sigma} T^{\rho \sigma}\right) \\
& -\frac{1}{m_{c}^{2}}\left(R_{\mu}^{\rho} R_{\rho \nu}-\frac{2}{3} R R_{\mu \nu}+\frac{1}{4} h_{\mu \nu} R^{2}-\frac{1}{2} h_{\mu \nu} R_{\rho \sigma} R^{\rho \sigma}\right) \\
& +\frac{\kappa_{4}^{2}}{m_{c}^{2}}\left(R_{\mu}^{\rho} T_{\rho \nu}+T_{\mu}^{\rho} R_{\rho \nu}-\frac{2}{3} R T_{\mu \nu}-h_{\mu \nu} R_{\rho \sigma} T^{\rho \sigma}\right),
\end{aligned}
$$

where we used $T=T_{\mu}^{\mu}=0$ and $m_{c}^{-1}=\kappa_{5}^{2} / 2 \kappa_{4}^{2}$. If we take the trace of (20), we get the Hamiltonian constraint (15). Note that the four-dimensional gravitational field equations (20) contains a term, $E_{\mu \nu}$, which carries information of the gravitational field outside the brane. In general, the field equations on the brane are not closed and one needs to solve the evolution equations into the bulk. However, by assuming a special ansatz for the induced metric on the brane, one can make the system of equations on the brane closed. 


\section{Charged black hole solutions}

For charged black holes on the brane, we assume the following spherically symmetric metric ansatz,

$$
\begin{aligned}
d s^{2} & =h_{\mu \nu} d x^{\mu} d x^{\nu} \\
& =-\left(1-\frac{P(r)}{r}\right) d t^{2}+\left(1-\frac{P(r)}{r}\right)^{-1} d r^{2}+r^{2} d \theta^{2}+r^{2} \sin ^{2} \theta d \phi^{2} .
\end{aligned}
$$

The Hamiltonian constraint (15) in the presence of a Maxwell field can be written as

$$
0=R_{\mu \nu} R^{\mu \nu}-\frac{1}{3} R^{2}+m_{c}^{2} R-2 \kappa_{4}^{2} R_{\mu \nu} T^{\mu \nu}+4 \kappa_{4}^{4} S
$$

where $S \equiv T_{\mu \nu} T^{\mu \nu} / 4=Q^{4} / 64 \pi^{2} r^{8}, T_{\mu \nu}=\left(F_{\mu \rho} F_{\nu}^{\rho}-\frac{1}{4} F_{\rho \sigma} F^{\rho \sigma}\right) / 4 \pi$.

We assume that the Maxwell field on the brane is described by a solution of source-free Maxwell equations. In this case, we must solve both the Hamiltonian constraint (22) and the Maxwell's equations:

$$
\begin{gathered}
g^{\mu \nu} D_{\mu} F_{\nu \sigma}=0, \\
D_{[\mu} F_{\nu \sigma]}=0 .
\end{gathered}
$$

Eq.(24) is satisfied identically if the field strength $F_{\mu \nu}$ is constructed by the potential oneform. One can easily verify that the solution of the Maxwell equations written in terms of a potential one-form under the metric ansatz (21) has the following form

$$
A=-\frac{Q}{r} d t
$$

where the parameter $\mathrm{Q}$ is thought of as the electric charge of the black hole. The energymomentum tensor for the above potential one-form $A$ is calculated to be

$$
T_{t t}=\frac{Q^{2}}{8 \pi r^{4}}(1-P / r), T_{r r}=-\frac{Q^{2}}{8 \pi r^{4}}(1-P / r)^{-1}, T_{\theta \theta}=\frac{Q^{2}}{8 \pi r^{2}}, T_{\phi \phi}=\frac{Q^{2} \sin ^{2} \theta}{8 \pi r^{2}} .
$$

Before solving the Hamiltonian constraint (22), we consider particular solutions of this constraint equation as a warm-up exercise. First, we notice that the following set of relations satisfy the constraint equation (22):

$$
R=12 m_{c}^{2}, R_{\mu \nu} R^{\mu \nu}=36 m_{c}^{4}+4 Q^{4} / r^{8}, R_{\mu \nu} T^{\mu \nu}=Q^{4} / 2 \pi r^{8} .
$$


These relations are satisfied, if the function $P$ in the metric ansatz (21) is given by

$$
P=-\frac{Q^{2}}{r}+m_{c}^{2} r^{3}+C
$$

where the parameter $C$ is an integration constant.

Now, we consider a general solution satisfying the Hamiltonian constraint equation with the metric ansatz (21). With the energy-momentum tensor (26) and the metric ansatz, the Hamiltonian constraint (22) can be expressed as

$$
0=6 m_{c}^{2} r^{2}\left(2 Z+r Z_{r}\right)+r^{2} Z_{r}^{2}-8 r Z Z_{r}+4 Z^{2}-\frac{12 Q^{2}}{r^{2}}\left(2 Z-r Z_{r}\right)+\frac{24 Q^{4}}{r^{4}}
$$

where $Z=d P / d r$ and $Z_{r}=d^{2} P / d r^{2}$. For $Q=0$, as one should expect, the above equation reduces to the equation for the Schwarzschild case [14]. To learn the behavior of exact solution, we first study asymptotic behaviors in two regions $r \gg r_{c}$ and $r \ll r_{c}$.

For $r \gg r_{c}$, we can neglect the last five terms on the right-hand side of (29) since the quadratic terms is viable only for lage distances. The solution in this case is given by

$$
P=\frac{\tilde{r}_{M}^{2}}{r}+C_{1} \text { for } r \gg r_{c}
$$

where $\tilde{r}_{M}$ and $C_{1}$ are integration constants. When $C_{1}=0$, this solution becomes the ordinary five-dimensional Schwarzschild solution of radius $\tilde{r}_{M}$.

For $r \ll r_{c}$, the first term on the right-hand side of (29) can be neglected and reduces to

$$
r^{2} Z_{r}^{2}-8 r Z Z_{r}+4 Z^{2}-\frac{12 Q^{2}}{r^{2}}\left(2 Z-r Z_{r}\right)+\frac{24 Q^{4}}{r^{4}} \approx 0
$$

Solving for $Z_{r}$, we get

$$
Z_{r}=-\frac{1}{r}\left[\frac{6 Q^{2}}{r^{2}}-4 Z \pm 2 \sqrt{3}\left(\frac{Q^{2}}{r^{2}}-Z\right)\right] .
$$

The solution for this is given by

$$
P=r_{M}-\frac{Q^{2}}{r}+C_{1} r^{5 \pm 2 \sqrt{3}} \text { for } r \ll r_{c}
$$

where $r_{M}$ and $C_{1}$ is integration constants. When $C_{1}=0$, this becomes the charged black hole solution for mass $r_{M} / 2$ and charge $Q$ in the general relativity. 
For an interpolating solution between these two regimes, we first solve (29) for $Z_{r}$ :

$$
Z_{r}=-\frac{1}{r}\left[\left(\frac{6 Q^{2}}{r^{2}}-4 Z\right)+3 m_{c}^{2} r^{2}\left(1 \pm \sqrt{1+4 u+\frac{4}{3} u^{2}}\right)\right]
$$

where $u=\left(Q^{2}-r^{2} Z\right) / m_{c}^{2} r^{4}$. Introducing a new function $U$ replacing $Z$,

$$
Z=Q^{2} / r^{2}-3 m_{c}^{2} r_{0}^{2} e^{2 z} U(z) / 2
$$

with $r=r_{0} e^{z}$, Eq.(34) can be reexpressed as

$$
-\frac{1}{r}\left[\frac{2 Q^{2}}{r^{2}}+\frac{3 m_{c}^{2} r^{2}}{2}\left(2 U+U_{z}\right)\right]=-\frac{1}{r}\left[\frac{2 Q^{2}}{r^{2}}+\frac{3 m_{c}^{2} r^{2}}{2}(4 U+2(1 \pm f))\right],
$$

where $f=\sqrt{1+6 U+3 U^{2}}$. One can now solve this equation in terms of $U_{z}$;

$$
U_{z}=2(1+U \pm f)
$$

Although the constraint equation (29) is different from the Schwarzschild case [14], we got the same equation (37) to solve. This equation has been solved implicitly and it has two solutions [14],

$$
\ln \left[-\frac{(1+3 U+f)}{F^{2}(3+3 U+\sqrt{3} f)^{2 \sqrt{3}}(-5-3 U+f)}\right]=8 z+C_{3}
$$

for the minus(-) sign in the last term in (37), and

$$
\ln \left[-\frac{(-5-3 U+f)(-3-3 U-\sqrt{3} f)^{2 \sqrt{3}}}{(U+2)^{2}(1+3 U+f)}\right]=8 z+C_{3}
$$

for the plus(+) sign, and $C_{3}$ is an integration constant.

Now, from $Z=d P / d r$ and (35), $P(r)$ can be determined by the following relation

$$
P(r)=-\frac{Q^{2}}{r}-\frac{3}{2} m_{c}^{2} \int d r r^{2} U(r)+C,
$$

where $C$ is an integration constant to be determined by the boundary condition. Note that Eq.(37) is satisfied with $U=-2$ for the plus sign and $U=0$ for the minus sign in (37), and the above relation for $P$ gives the same particular solution that we obtained in (28). 
Finally, using the relation $r=r_{0} e^{z}$, we can reexpress (38) and (39) in terms of $r$ as follows [21,

$$
\begin{aligned}
& \left(k_{1} r\right)^{8}=-\frac{(1+3 U+f)}{U^{2}(3+3 U+\sqrt{3} f)^{2 \sqrt{3}}(-5-3 U+f)} \\
& \left(k_{2} r\right)^{8}=-\frac{(-5-3 U+f)(-3-3 U-\sqrt{3} f)^{2 \sqrt{3}}}{(U+2)^{2}(1+3 U+f)}
\end{aligned}
$$

where the two integration constant $k_{1}$ and $k_{2}$ are determined by imposing appropriate boundary conditions. The above two solutions are the so-called regular and accelerated branches, respectively [14].

For the regular branch solution (41) we impose the boundary conditions, $P(r)+Q^{2} / r \rightarrow$ $r_{M}$ for $r \rightarrow 0$ and $P(r) \rightarrow 0$ for $r \rightarrow \infty$. The integration constant $k_{1}$ can be obtained from (401) and is given by [21]

$$
2\left(k_{1} r_{*}\right)^{3} \equiv c \approx 0.43
$$

where $r_{*}=\left(r_{M} r_{c}^{2}\right)^{1 / 3}$. Here $\mathrm{c}$ is given by the following integral

$$
c=\int_{0}^{\infty} d U\left[-\frac{(1+3 U+f)}{U^{2}(3+3 U+\sqrt{3} f)^{2 \sqrt{3}}(-5-3 U+f)}\right]^{3 / 8} .
$$

We can easily see the following asymptotic behavior by consulting the result of [21]. At large distances, $r \gg r_{*}\left(U \rightarrow 0^{+}\right)$, (41) is approximated as

$$
U \approx \frac{\sqrt{2}}{2(3+\sqrt{3})^{\sqrt{3}}\left(k_{1} r\right)^{4}} .
$$

Using (43) and (45) one obtains

$$
\frac{P(r)}{r} \approx \frac{\tilde{r}_{M_{1}}^{2}-Q^{2}}{r^{2}}
$$

where $\tilde{r}_{M_{1}}^{2}=\frac{3 \sqrt{2}}{4(3+\sqrt{3})^{\sqrt{3}}} \frac{m_{c}^{2}}{k_{1}^{4}} \approx 0.56 r_{M} r_{*}$.

In the regular branch solution, there appeared the screened mass effect in the Schwarzschild case [14, 21]. The same effect appears here, too. However, there is no such effect for the charge, as one can expect from the form of our solution (40).

At short distances, $r \ll r_{*}(U \rightarrow+\infty)$, (41) is approximated as

$$
U \approx 6^{\frac{\sqrt{3}-3}{2}}\left(\frac{3+\sqrt{3}}{3-\sqrt{3}}\right)^{\frac{\sqrt{3}-1}{4}}\left(k_{1} r\right)^{2(1-\sqrt{3})} .
$$


Using (43) and (47) we obtain

$$
\frac{P(r)}{r} \approx \frac{r_{M}}{r}-\frac{Q^{2}}{r^{2}}-\alpha_{1} m_{c}^{2} r^{2}\left(\frac{r_{*}}{r}\right)^{2(\sqrt{3}-1)}
$$

where $\alpha_{1} \approx 0.84[21$.

The accelerated branch solution is obtained from the solution of (42) by imposing the boundary condition $P(r)+Q^{2} / r \rightarrow r_{M}$ for $r \rightarrow 0$ and $P(r)-m_{c}^{2} r^{3} \rightarrow 0$ for large $r$. In order to determine the integration constant $k_{2}$, imposing the above boundary condition at the (40), we obtain $k_{2}$ from the following relation [21]

$$
2\left(k_{2} r_{*}\right)^{3}=c^{\prime} \approx 4.41,
$$

where $c^{\prime}$ is obtained from the following integral

$$
c^{\prime}=\int_{-\infty}^{-2}\left[-\frac{(-5-3 U+f)(-3-3 U-\sqrt{3} f)^{2 \sqrt{3}}}{(U+2)^{2}(1+3 U+f)}\right]^{3 / 8} d U
$$

One can see the following asymptotic behavior by consulting the result of [21].

At large distances, $r \gg r_{*}\left(U \rightarrow-2^{-}\right)$, (42) is approximated as

$$
U+2 \approx-\frac{\sqrt{2}(3-\sqrt{3})^{\sqrt{3}}}{2\left(k_{2} r\right)^{4}}
$$

Using (49) and (51) one obtains

$$
\frac{P(r)}{r} \approx-\frac{\tilde{r}_{M_{2}}^{2}+Q^{2}}{r^{2}}+m_{c}^{2} r^{2}
$$

where $\tilde{r}_{M_{2}}^{2}=6^{\sqrt{3}} \frac{3 \sqrt{2}}{4(3+\sqrt{3})^{\sqrt{3}}} \frac{m_{c}^{2}}{k_{2}^{4}}=\frac{3 \sqrt{2}}{4(3+\sqrt{3})^{\sqrt{3}}} \frac{m_{c}^{2}}{k_{1}^{4}} \approx 0.56 r_{M} r_{*}$.

As in the regular branch case the screened mass effect occurs, but the screened charge effect does not occur in the accelerated branch case also.

At short distances, $r \ll r_{*}(U \rightarrow-\infty)$, since $f \approx-\sqrt{3}(1+U+1 / 6 U)$, (42) is approximated as

$$
U \approx-2^{\frac{\sqrt{3}-3}{2}}\left(\frac{3+\sqrt{3}}{3-\sqrt{3}}\right)^{\frac{\sqrt{3}-1}{4}}\left(k_{2} r\right)^{2(1-\sqrt{3})} .
$$

Using (49) and (53) one obtains

$$
\frac{P(r)}{r} \approx \frac{r_{M}}{r}-\frac{Q^{2}}{r^{2}}-\alpha_{2} m_{c}^{2} r^{2}\left(\frac{r_{*}}{r}\right)^{2(\sqrt{3}-1)},
$$

where $\alpha_{2}=-\alpha_{1} \approx 0.84[21$. 


\section{Conclusion}

In this paper we study charged black holes on the brane in the DGP model. Beginning with an ansatz for the induced metric on the brane, we solve the constraint equations of $(4+1)$-dimensional gravity to find metrics describing charged brane-world black holes. In the absence of the Maxwell charge, we obtain the Schwarzschild solution obtained previously by Gabadadze and Iglesias. In the presence of the Maxwell charge, we obtain charged black hole solutions which are the Reissner-Nordstrom type with some corrections. One type of our solutions exhibits the phenomena of the accelerated expansion, the same behavior as in the Schwarzschild case. As in the Schwarzschild case, the screened mass effect also occurs in the charged case. However, no such effect for the charge appears.

\section{Acknowledgments}

This work was supported by the Korea Research Foundation Grant funded by the Korean Government(MOEHRD), KRF-2006-312-C00498. E. C.-Y. thanks KIAS for hospitality during the time that this work was done.

\section{References}

[1] S. Perlmutter et al., Astrophys. J. 517 (1999) 565.

[2] G. Dvali, G. Gabadadze, and M. Porrati, Phys. Lett. B 485 (2000) 208.

[3] C. Deffayet,Phys. Lett. B 502 (2001) 199; C. Deffayet, G. Dvali, and G. Gabadadze, Phys. Rev. D 65 (2002) 044023.

[4] L. Randall and R. Sundrum, Phys. Rev. Lett. 83 (1999) 3370; 83 (1999) 4690.

[5] A. Chamblin, S. W. Hawking, and H. S. Reall, Phys. Rev. D 61 (2000) 065007.

[6] N. Dadhich, R. Maartens, P. Papadopoulos, V. Rezania, Phys. Lett. B 487 (2000) 1.

[7] A. Chamblin, H. S. Reall, H. Shinkai, and T. Shirmizu, Phys. Rev. D 63 (2001) 064015. 
[8] A. N. Aliev and A. E. Gumrukcuoglu, Phys. Rev. D 71 (2005) 104027.

[9] M. Porrati, Phys. Lett. B 534 (2002) 209.

[10] G. Kofinas, E. Papantonopoulos, and I. Pappa, Phys. Rev. D 66 (2002) 104014; G. Kofinas, E. Papantonopoulos, and V. Zamarias, Phys. Rev. D 66 (2002) 104028.

[11] A. Lue and G. Starkman, Phys. Rev. D 67 (2003) 064002.

[12] C. Middleton and G. Siopsis, Mod. Phys. Lett. A 19 (2004) 2259.

[13] A. Nicolis and R. Rattazzi, JHEP 0406 (2004) 059.

[14] G. Gabadadze and A. Iglesias, Phys. Rev. D 72 (2005) 084024.

[15] M. Kolanovic, Phys. Rev. D 65 (2002) 124005.

[16] A. N. Aliev and A. E. Gumrukcuoglu, Class. Quantum Grav. 21 (2004) 5081.

[17] G. W. Gibbons and S. W. Hawking, Phys. Rev. D 15 (1977) 2738.

[18] C. Deffayet, G. Gabadadze and A. Iglesias, JCAP 0608 (2006) 012.

[19] W. Israel, Nuovo. Cim. B 44 (1966) 1; 48 (1967) 463(E).

[20] T. Shiromizu, K. Maeda, and M. Sasaki, Phys. Rev. D 62 (2000) 024012.

[21] G. Gabadadze and A. Iglesias, Phys. Lett. B 632 (2006) 617. 